\title{
Speed-dependent adaptive partitioning QM/MM for displacement damage simulations
}

\author{
Zeng-hui Yang*, $\dagger$ \\ Microsystem and Terahertz Research Center, China Academy of Engineering Physics, Chengdu, \\ China 610200, and Institute of Electronic Engineering, China Academy of Engineering Physics, \\ Mianyang, China 621000 \\ E-mail: yangzenghui@mtrc.ac.cn
}

\section{Abstract}

Solids receive displacement damages (DD) when interacting with energetic particles, which may happen during the fabrication of semiconductor devices, in harsh environments and in certain analysis techniques. Simulations of the DD generation are usually carried out with classical molecular dynamics (MD), but classical MD does not account for all the effects in DD, as demonstrated by $a b$ initio calculations of model systems in literature. A fully $a b$ initio simulation of the DD generation is impractical due to the large number of atoms involved. In my previous paper [Phys. Chem. Chem. Phys. 22, 19307 (2020)], I developed an adaptive-center (AC) method for adaptivepartitioning (AP) quantum mechanics/molecular mechanics (QM/MM) simulations, allowing the active region centers and the QM/MM partition to be determined on-the-fly for energy-conserving AP-QM/MM methods. I demonstrated that the AC-AP-QM/MM is applicable to the simulation of the DD generation, so that the active region$\mathrm{s}$ can be treated with an $a b$ initio method. The $\mathrm{AC}$ method was unable to identify the fast-moving recoil ions in the DD generation as active region centers, however, and the accuracy is negatively affected by the rapid change in QM/MM parti-

\footnotetext{
*To whom correspondence should be addressed

${ }^{\dagger}$ Microsystem and Terahertz Research Center, China Academy of Engineering Physics, Chengdu, China 610200

Institute of Electronic Engineering, China Academy of Engineering Physics, Mianyang, China 621000
}

tion of the system. In this paper, I extend the AC method and develop a speed-dependent adaptivecenter (SDAC) method for proper AP-QM/MM simulations of DD. The SDAC method is applicable to general problems with speed-dependent active regions, and is compatible with all existing energy-conserving partition-by-distance APQM/MM methods. The artifact due to the speeddependent potential energy surface can be made small by choosing proper criteria. I demonstrate the SDAC method by simulations of the DD generation in bulk Silicon.

\section{Introduction}

Displacement damages (DD) in solid occur as the material interacts with impinging energetic particles, during which atoms are dislodged from their original sites by the incident particle or recoil ions, generating both isolated defects and disordered regions due to collision cascades. ${ }^{1-3}$ DD is produced in a wide range of applications, such as in radiative environments including nuclear reactors, accelerators and the space, in the fabrication of semiconductor devices with ion implantation and ion beam deposition technologies, and in ion-beam related analysis techniques such as the secondary ion mass spectroscopy. Direct experimental measurements of the DD generation have been difficult due to the small spatial and temporal scales, and much effort has been devoted to simulation studies. ${ }^{4-35}$

A large number of atoms are needed for simulating the DD generation, since its collision cascades 
may span several nanometers. ${ }^{1,2,16}$ The number of atoms exceeds the ability of highly accurate $a b i$ nitio quantum mechanical (QM) methods such as the density functional theory (DFT). ${ }^{36-38}$ More computationally efficient methods such as the binary collision approximation (BCA) ${ }^{39-42}$ and the molecular dynamics (MD) with classical molecular mechanics (MM) potentials are therefore dominant in simulation studies of the DD. Both methods have limitations: BCA cannot describe collective many-body effects, $4,5,17,18$ and its Frenkelpair based description of the DD becomes inappropriate for the amorphous disordered regions; ${ }^{4,18,19}$ classical MD is less accurate for the far-fromequilibrium geometries during collision cascades, and does not fully account for the changes in the electronic structure. ${ }^{6,17,20-22,43}$ The deficiencies of these methods in simulating the DD can be seen in the QM studies of model systems, ${ }^{20-23}$ which reveal effects and processes in the DD that were missing from BCA and classical MD simulations. A more clear demonstration of the deficiencies is recently provided by Hamedani, et al, ${ }^{14}$ in which a DFT-trained machine-learning potential is shown to yield highly different DD results from those of commonly used classical potentials.

Quantum mechanics/molecular mechanics (QM/MM) methods ${ }^{44-50}$ combine the accuracy and explicit treatment of electrons of QM methods and the efficiency of classical MM potentials, and it should be able to cure the aforementioned deficiencies of the BCA and the classical MD while avoiding the overwhelming computational cost of pure QM simulations. QM/MM methods partition the simulated system into small active (QM) regions and large environmental (MM) regions, treat the atoms in QM and MM regions with higherlevel and lower-level methods respectively, and couple the QM and MM parts together. For the simulation of DD, the active regions would consist of the regions in which classical MM potentials become less accurate, such as the regions around recoil ions (including the primary knock-on atom, the PKA) due to their strong disturbance to the local electronic structure, and the disordered regions with far-from-equilibrium geometries. Both types of regions would occur or vanish dynamically during the simulation, disqualifying the application of regular QM/MM methods in which the partition of the system is determined beforehand and kept fixed.

Adaptive-partitioning (AP) QM/MM ${ }^{49-61}$ extends QM/MM and allows the partition of the system to change during the MD simulation. Most of the energy-conserving AP-QM/MM method$\mathrm{s}$ partition the system according to distances to active region centers. ${ }^{54,55,57,58}$ The active region centers are chosen beforehand and kept fixed during the simulation, which is unsuitable for the simulation of the DD. Recently, I developed an adaptive-center (AC) method ${ }^{62}$ which allows active region centers to change on-the-fly according to any geometrical criterion, and carried out a proof-of-concept simulation of the DD using the virial stress per atom to identify the disordered regions. The regions with far-from-equilibrium geometries are treated with QM in the AC simulation as expected, but the AC method fails to identify the regions around the fast-moving recoil ions as QM regions, since it would require a speed-dependent criterion. Aside from this problem, it is observed that the sizes of the QM regions change rapidly in the AC simulations, ${ }^{62}$ which hampers the accuracy of the simulation.

In this paper, I develop a speed-dependent adaptive-center (SDAC) partitioning method for carrying out proper adaptive-partitioning (AP) ${ }^{49-61} \mathrm{QM} / \mathrm{MM}$ simulations of the DD. The method is (a) compatible with all the existing energy-conserving AP-QM/MM methods that partition the system by distances to active sites, ${ }^{51-55,57,58,63,64}$ (b) able to determine active region centers according to any atomic property that is fully determined by the positions and speeds of atoms, and (c) yields a smooth potential energy surface (PES) by construction. I derive the non-Lagrangian equation of motion (EOM) for the speed-dependent PES, and show that the artifact of the EOM can be negligible with properly chosen criterion. I carry out SDAC-AP-QM/MM simulations of the DD in Silicon to showcase the power of the method. 


\section{Method}

\subsection{Adaptive partitioning by distance with adaptive centers}

The QM/MM partitioning is determined in most AP-QM/MM methods according to distances to active region centers ${ }^{51-58,63,64}$ as illustrated in Fig. 1. For each atom, one calculates a weight according to its distances to the centers. The weight is 0,1 or in the $(0,1)$ range when the atom belongs to the MM, QM or buffer region. For atom $\alpha$, its weight $\lambda_{\alpha}$ is calculated according to

$$
\lambda_{\alpha}=1-\prod_{\zeta}^{\text {Centers }}\left[1-f\left(\tilde{\lambda}_{\alpha, \zeta}\right)\right]
$$

where

$$
f\left(\tilde{\lambda}_{\alpha, \zeta}\right)=10 \tilde{\lambda}_{\alpha, \zeta}^{3}-15 \tilde{\lambda}_{\alpha, \zeta}^{4}+6 \tilde{\lambda}_{\alpha, \zeta}^{5},
$$

and

$$
\begin{aligned}
& \tilde{\lambda}_{\alpha, \zeta}=\left(1-\frac{R_{\alpha, \zeta}-R_{\zeta}^{\mathrm{QM}}}{W_{\zeta}^{\mathrm{buf}}}\right) \theta\left(R_{\alpha, \zeta}-R_{\zeta}^{\mathrm{QM}}\right) \\
& \quad \times \theta\left(R_{\zeta}^{\mathrm{QM}}+W_{\zeta}^{\mathrm{buf}}-R_{\alpha, \zeta}\right)+\theta\left(R_{\zeta}^{\mathrm{QM}}-R_{\alpha, \zeta}\right),
\end{aligned}
$$

with $\theta$ being the Heaviside step function, $R_{\alpha, \zeta}=$ $\left|\vec{R}_{\alpha}-\vec{R}_{\zeta}\right|$ being the distance between atom $\alpha$ and center $\zeta, R_{\zeta}^{\mathrm{QM}}$ and $W_{\zeta}^{\text {buf }}$ being the radius of the QM region and the thickness of the buffer region associated with center $\zeta$. Although different APQM/MM methods use the atomic weights $\lambda$ of Eq. (1) differently, all treat the buffer atoms with both the QM and the MM methods, and the weight of a buffer atom represents the percentage of its QM character.

The AC method ${ }^{62}$ determines whether an atom is an active region center according to any atomic property (denoted as $\xi$ ), with the restriction that $\xi$ can be fully determined by atomic positions. $R_{\zeta}^{\mathrm{QM}}$

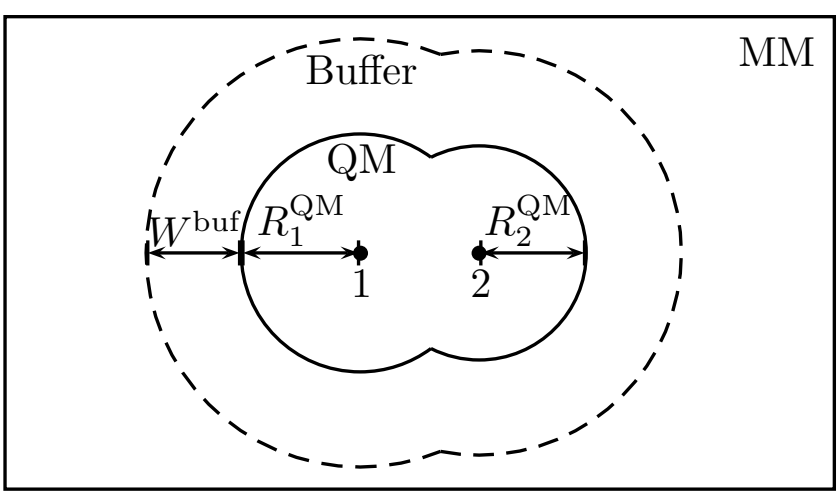

Figure 1: Illustration of the AP-QM/MM partition of the system according to distances to active region centers. Two active region centers (1 and 2) are drawn in the figure.

in the AC method is calculated by

$$
\begin{aligned}
& R_{\zeta}^{\mathrm{QM}}=R_{\max }^{\mathrm{QM}}\left\{\theta\left(\xi_{\zeta}-\xi_{\max }^{\mathrm{QM}}\right)+\theta\left(\xi_{\max }^{\mathrm{QM}}-\xi_{\zeta}\right)\right. \\
\times & {\left.\left[\theta\left(\xi_{\zeta}-\xi_{\min }^{\mathrm{semi}}\right) f\left(\xi_{\zeta}\right)+\theta\left(\xi_{\min }^{\mathrm{semi}}-\xi_{\zeta}\right) f\left(\xi_{\min }^{\mathrm{semi}}\right)\right]\right\} }
\end{aligned}
$$

where $f$ is a smoothing function, ${ }^{62} R_{\max }^{\mathrm{QM}}, \xi_{\min }^{\text {semi }}$, $\xi_{\min }^{\mathrm{QM}}, \xi_{\max }^{\mathrm{QM}}$ are the $\mathrm{AC}$ partition parameters satisfying $\xi_{\min }^{\text {semi }}<\xi_{\min }^{\mathrm{QM}}<\xi_{\max }^{\mathrm{QM}}$. $W^{\text {buf }}$ of all centers are calculated by

$$
W^{\text {buf }}=-\left.R_{\zeta}^{\mathrm{QM}}\right|_{\xi_{\zeta}=\xi_{\min }^{\mathrm{semi}}} .
$$

An atom $\zeta$ is an active region center if $R_{\zeta}^{\mathrm{QM}}>0$, is a regular atom if $R_{\zeta}^{\mathrm{QM}}+W^{\text {buf }} \leq 0$, and is denoted as a semi-center if $R_{\zeta}^{\mathrm{QM}} \leq 0$ and $R_{\zeta}^{\mathrm{QM}}+W^{\text {buf }}>$ 0 . A semi-center only has associated buffer region but no QM region.

The transition forces in AP-QM/MM are the weights' contribution to the forces. They have the following form in the $\mathrm{AC}$ method:

$$
\vec{F}_{\alpha}^{\mathrm{tr}}=-\sum_{\beta}\left[\frac{\partial V}{\partial \lambda_{\beta}}\left(\nabla_{\vec{R}_{\alpha}} \lambda_{\beta}+\sum_{\gamma} \frac{\partial \lambda_{\beta}}{\partial \xi_{\gamma}} \nabla_{\vec{R}_{\alpha}} \xi_{\gamma}\right)\right],
$$

where $V$ is the QM/MM potential energy (PE) defined as

$$
V=V^{\mathrm{QM}}+V^{\mathrm{MM}}+V^{\mathrm{int}},
$$

with $V^{\mathrm{QM}}$ and $V^{\mathrm{MM}}$ being the potential energies of 
the QM and MM subsystems respectively, and $V^{\text {int }}$ being the interaction energy between the QM and MM subsystems.

The atomic weights $\lambda$ and the criterion $\xi$ are completely determined by atomic positions, so the PE of Eq. (7) is also completely determined by atomic positions in the AC method. The total energy is equal to the Hamiltonian for such a $\mathrm{PE}^{65}$ and is conserved in microcanonical (NVE) simulations with the Newton's second law EOM derived from the Lagrangian dyanmics.

\subsection{The SDAC equation of motion}

I develop the SDAC method by extending the AC method to allow speed-dependent criterion, so as to carry out QM/MM simulations of the DD generation in solids. Such simulations are carried out in the NVE ensemble with temperature controlling layers, ${ }^{4-15}$ so I only discuss NVE simulations in the following. A speed-dependent $\xi$ results in a PES that depends on both the atomic positions and the speeds, and the Newton's second law EOM no longer applies. The Hamiltonian of the system becomes 65

$$
H=T+V-\sum_{\alpha} \dot{\vec{r}}_{\alpha} \cdot \nabla_{\dot{\vec{r}}_{\alpha}} V
$$

where $T$ and $V$ are the kinetic and QM/MM potential energies respectively, $\vec{r}_{\alpha}$ is the position of atom $\alpha$, and the dot in $\dot{\vec{r}}_{\alpha}$ represents taking the time derivative.

Eq. (8) describes the dynamics of the artificially constructed SDAC-AP-QM/MM system instead of that of the real system, however, as the NVE EOM derived from Lagrangian dynamics (Appendix A) conserves the Hamiltonian of Eq. (8) instead of the total energy $E=T+V .{ }^{65}$ Since the purpose of simulations is to study the real system, I derive the following energy-conserving SDAC EOM starting from $\dot{E}=0$ :

$$
\left(m_{\alpha}+\frac{1}{v_{\alpha}} \frac{\partial V}{\partial v_{\alpha}}\right) \vec{a}_{\alpha}=-\nabla_{\vec{r}_{\alpha}} V
$$

where $m_{\alpha}$ is the mass of atom $\alpha, v_{\alpha}=\left|\dot{\vec{r}}_{\alpha}\right|$ is the speed of atom $\alpha$, and $\vec{a}_{\alpha}=\ddot{\vec{r}}_{\alpha}$ is the acceleration of atom $\alpha . \partial V / \partial v_{\alpha}$ is

$$
\frac{\partial V}{\partial v_{\alpha}}=\sum_{\beta} \frac{\partial V}{\partial \lambda_{\beta}} \sum_{\gamma} \frac{\partial \lambda_{\beta}}{\partial \xi_{\gamma}} \frac{\partial \xi_{\gamma}}{\partial v_{\alpha}} .
$$

The speed-dependent effective mass (SDEM) $(1 / v)\left(\partial V / \partial v_{\alpha}\right)$ in Eq. (9) is an artifact of the SDAC method. It is the side-effect of smoothly joining the QM PES and the MM PES with atomic weights Eq. (1), similar to the transition forces of Eq. (6). Transition forces lead to geometry distortions, ${ }^{55,66,67}$ and the SDEM also lead to deviations from the real dynamics. Transition forces can be corrected by adding compensating terms to the Hamiltonian ${ }^{54,55,67}$ or by dropping it directly in simulations coupled to a thermostat. ${ }^{66}$ The SDEM can be treated similarly either by developing a Hamiltonian correction or by discarding the term. For AP-QM/MM methods that mix the results of many QM and MM calculations ${ }^{54-57}$ (referred to as multi-pass methods hereafter), it is straightforward to develop Hamiltonian corrections of transition forces, ${ }^{54,55,67}$ and the same goes for the correction of the SDEM.

For simulations of the DD generation, the computational cost of the multi-pass methods can be overwhelming. ${ }^{62,68}$ I use the mod-SISPA method of my previous work ${ }^{68}$ in this paper, which only need one QM and one MM calculations for each time step. For such 'single-pass' AP-QM/MM methods, ${ }^{58,68}$ corrections to the transition forces and the SDEM are unavailable and difficult to develop. Since most DD simulations are done in the NVE ensemble, ${ }^{4-15}$ neglecting the SDEM is inapplicable as well. I therefore do not apply corrections to the SDEM for the SDAC simulations in this paper. This is reasonable if the SDEMs are orders of magnitudes smaller than $m_{\alpha}$, which would ensure the dynamics closely follow that of the real system. In Sec. 3.2, I show that a properly chosen functional form for $\xi$ can effectively reduce the size of the SDEM.

The velocity Verlet algorithm ${ }^{69,70}$ is the most common time integration algorithm in MD simulations, but it is inapplicable to the integration of Eq. (9) due to its assumption of velocity-independent acceleration. The extended phase space (EPS) algorithm $^{71-74}$ is an explicit time integration algorithm applicable to non-Lagrangian EOMs such 
as Eq. (9). It extends the initial phase space $\left(\left\{\vec{r}_{\alpha}\right\},\left\{\vec{v}_{\alpha}\right\}\right)$ with its duplicate $\left(\left\{\tilde{\vec{r}}_{\alpha}\right\},\left\{\tilde{\vec{v}}_{\alpha}\right\}\right)$ at the initial time, and carries out a leapfrog-type time integration ${ }^{75}$ in the EPS. The EPS algorithm does not show accumulation of numerical error in the total energy, despite being symplectic ${ }^{70}$ only in the EPS and not in the original phase space. ${ }^{71} \mathrm{I}$ use the EPS algorithm for all time integrations of Eq. (9) in this paper. Details of the EPS algorithm are available in Appendix B.

\section{Results}

I demonstrate the SDAC method by simulations of the DD generation in bulk Silicon. The number of QM calculations involved in the multi-pass AP-QM/MM methods ${ }^{54-57}$ is at least the number of buffer atoms, which is too high for DD simulations. I use the mod-SISPA AP-QM/MM method of my previous work ${ }^{68}$ which only requires one QM calculation per PE evaluation. Since a purely QM calculation of the entire system is impractical, I check the energy conservation of SDAC with $\mathrm{MM} / \mathrm{MM}$ calculations. MM/MM calculations are also carried out to verify the SDAC method against a purely MM calculation.

I use the density-functional tight-binding (DFTB) method ${ }^{76-78}$ for the QM calculation and the Stillinger-Weber (SW) potential ${ }^{79}$ as the MM method in QM/MM simulations. The Tersoff potential ${ }^{80}$ with the Ziegler-Biersack-Littmark (ZBL) short-range interaction ${ }^{41}$ (Tersoff/ZBL) is used as the 'higher-level' method for the MM/MM simulations in Sec. 3.2.

I implement the SDAC method in the LAMMP$S^{81,82}$ code, and the DFTB calculations in modSISPA are carried out with a modified version of the DFTB $+{ }^{83}$ code. The OVITO software ${ }^{84}$ is used for the visualization of the results.

\subsection{SDAC criterion using full per- atom stress}

In the $\mathrm{AC}$ simulations of my previous work, ${ }^{62}$ the criterion $\xi^{\text {virial }}$ for the proof-of-concept DD simulations is

$$
\xi_{\alpha}^{\text {virial }}=\log _{10}\left|V_{\alpha}\left(\sigma_{\mathrm{V}, \alpha}^{x x}+\sigma_{\mathrm{V}, \alpha}^{y y}+\sigma_{\mathrm{V}, \alpha}^{z z}\right)\right|,
$$

where $V_{\alpha}$ is the volume of atom $\alpha$, and $\stackrel{\leftrightarrow}{\sigma}_{\mathrm{V}, \alpha}$ is the virial contribution to the per-atom stress tensor of atom $\alpha$ :

$$
\stackrel{\leftrightarrow}{\sigma}_{\mathrm{V}, \alpha}=\frac{\vec{r}_{\alpha} \otimes \vec{F}_{\alpha}}{V_{\alpha}},
$$

with $\otimes$ representing tensorial direct product, $\vec{r}_{\alpha}$ and $\vec{F}_{\alpha}$ being the position and the force of $\alpha$ respectively. To simplify the implementation, I evaluate $\vec{F}_{\alpha}$ of Eq. (12) with the Lennard-Jones potential with the OpenKIM parametrization. ${ }^{85-88}$

$\stackrel{\leftrightarrow}{\sigma}_{\mathrm{V}, \alpha}$ is fully determined by the geometry of the system. Since $\xi_{\alpha}$ virial is the smallest at equilibrium geometry, it is able to identify the regions with far-from-equilibrium geometry as QM regions. It can be argued that the regions around the fastmoving recoil ions should have higher priority for the QM treatment, however, since the electronic stopping power ${ }^{41,42,89,90}$ and the electron-phonon coupling can influence the range of the ion and the shape of the cascade. ${ }^{90} \xi^{\text {virial }}$ only identifies the fast-moving recoil atoms as active region centers when they are about to collide with other atoms as demonstrated in Fig. 2, leading to an inconsistent description of the electronic effects ${ }^{1-3,90,91}$ of the DD.

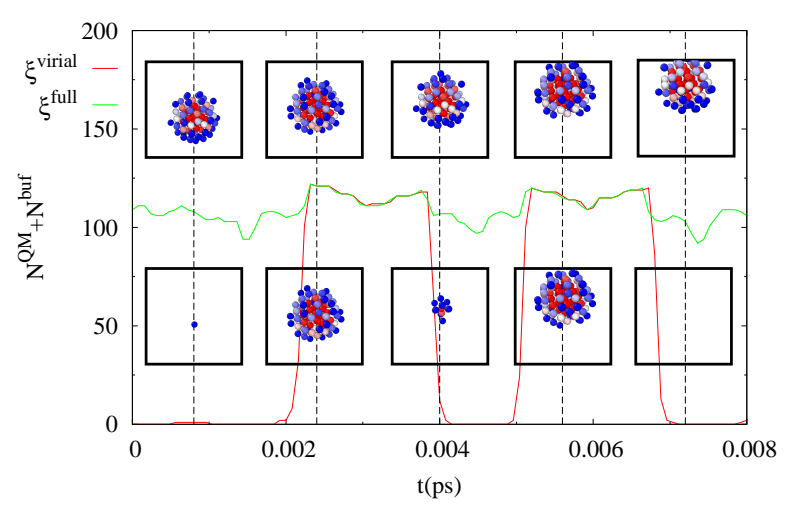

Figure 2: The number of QM and buffer atoms determined by the AC ( $\xi^{\text {virial }}$ curve) and the SDAC ( $\xi^{\text {full }}$ curve) methods in a MD simulation of the D$\mathrm{D}$ generation with the Tersoff/ZBL potential. The insets show snapshots of the QM and buffer atoms, and the color coding indicates the atomic weights (blue-white-red representing $\lambda=0$ to $\lambda=1$ ). The upper insets are snapshots of the SDAC simulation, and the lower insets are snapshots of the AC simulation.

For the SDAC simulations in this paper, I use 
$\xi$ full as the criterion:

$$
\xi_{\alpha}^{\text {full }}=\log _{10}\left|V_{\alpha}\left(\sigma_{\alpha}^{x x}+\sigma_{\alpha}^{y y}+\sigma_{\alpha}^{z z}\right)\right|
$$

where the full per-atom stress $\stackrel{\leftrightarrow}{\sigma}_{\alpha}$ is

$$
\stackrel{\leftrightarrow}{\sigma}_{\alpha}=\frac{m_{\alpha} \vec{v}_{\alpha} \otimes \vec{v}_{\alpha}+\vec{r}_{\alpha} \otimes \vec{F}_{\alpha}}{V_{\alpha}}
$$

with $\vec{v}_{\alpha}$ being the velocity of atom $\alpha$. The SDAC method identify both the regions around recoil ion$\mathrm{s}$ and with the distorted geometries as QM region-

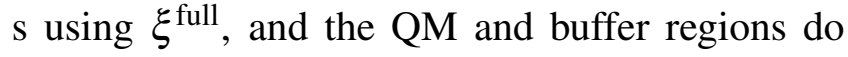
not change as rapidly as the AC simulations using $\xi^{\text {virial }}$ (Fig. 2).

\subsection{Validation of the SDAC method}

I validate the SDAC EOM Eq. (9) by comparing SDAC MM/MM results with those calculated with the 'lower-level' (SW) and the 'higher-level' (Tersoff/ZBL) MM method. The active regions and the atoms inside are still referred to as QM regions and $\mathrm{QM}$ atoms for these MM/MM simulations.

\subsubsection{Conservation and the SDEM}

I check the energy and momentum conservation of the SDAC method with NVE simulations. The simulations are carried out with an $17 \times 17 \times 17$ bulk Si supercell equilibrated at $300 \mathrm{~K}$ with periodic boundary conditions. I use a variable time step so that max allowed distance for an atom to move in one time step is $0.002 \AA$, and the max time step is 1 fs. The lattice constant is fixed at $5.43 \AA$ to ensure that the results of different methods are directly comparable. The Si atom at the center of the simulation box is chosen as the PKA. The initial kinetic energy of the PKA is set to $1 \mathrm{keV}$, and the initial velocity is $7^{\circ}$ away from the $z$ direction to avoid channelling. ${ }^{10,34}$ The results are shown in Fig. 3. An AC calculation with $\xi^{\text {virial }}$ as criterion and MM calculations with Tersoff/ZBL and SW potentials are carried out for comparison, and the velocity Verlet algorithm is used for their time integration.

Fig. 3(a) shows that the numerical errors in the SDAC total energies are at the same orders of magnitude as those in the AC and MM total energies. The SDAC total energy errors do not accumulate

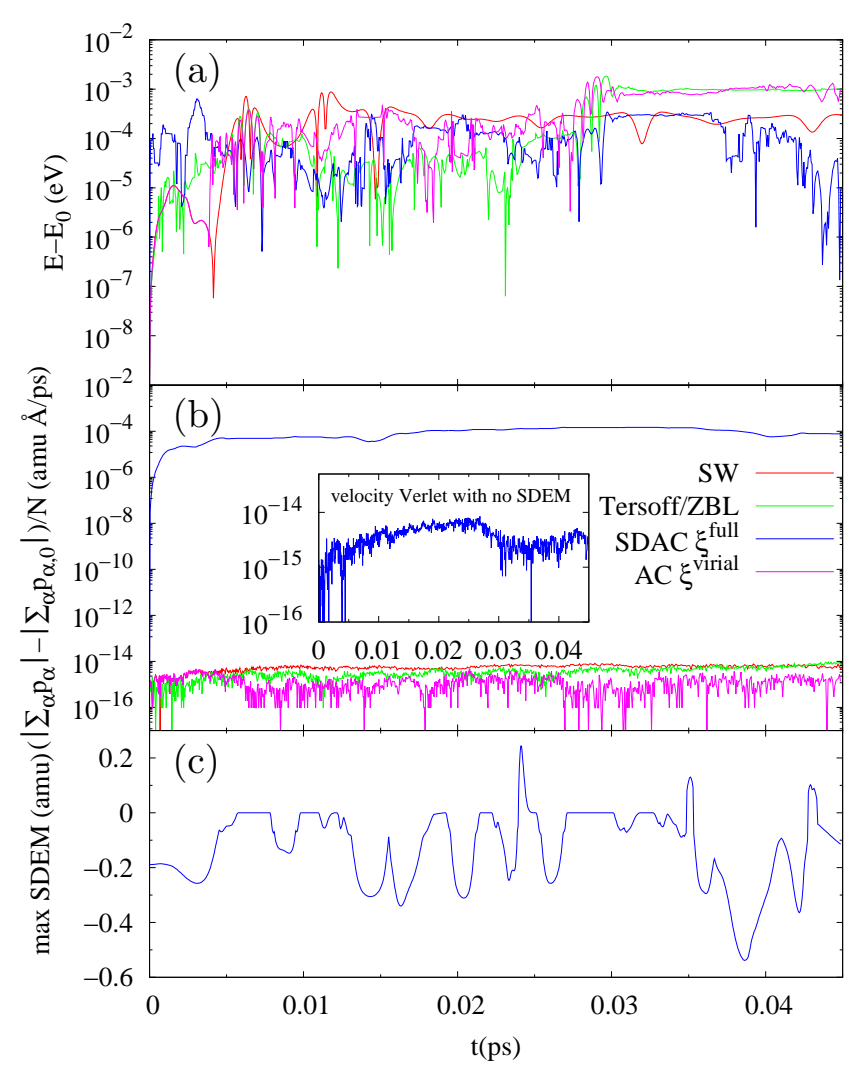

Figure 3: The energy and momentum conservation of the SDAC method in NVE simulations. Panel (a) plots the changes in the total energies with respect to time. Panel (b) plots the per-atom change of the total momentum with respect to time. The SDEMs with the largest absolute value are plotted in panel (c) for comparison. The partition parameters are $R_{\max }^{\mathrm{QM}}=4 \AA$, $\xi_{\min }^{\mathrm{semi}}=8, \xi_{\min }^{\mathrm{QM}}=9$, and $\xi_{\max }^{\mathrm{QM}}=10$ for the AC and the SDAC simulations. 
over time, which verifies the stability of the EPS algorithm applied on the EOM Eq. (9). The energy conservation is ensured by the EOM and is not affected by the changes in the SDEM.

Fig. 3(b) plots the numerical error in the total momentum per atom comparing with the value at the initial time, which measures the deviation from momentum conservation. The momentum is conserved in the MM and AC simulations. The SDAC appears to be worse in this aspect with much larger errors than those of the MM and the AC simulations. The inset of Fig. 3(b) plots the SDAC total momentum errors when using velocity Verlet time integration. Such simulations do not conserve the total energy, but the total momentum errors are at the same orders of magnitudes as the MM and the AC simulations. This shows that the deviation from momentum conservation of the SDAC curves is purely numerical due to the EPS time integration, since the actual time propagation happens in the extended phase space, but the curves plotted in Fig. 3(b) are obtained by projection (Appendix B).

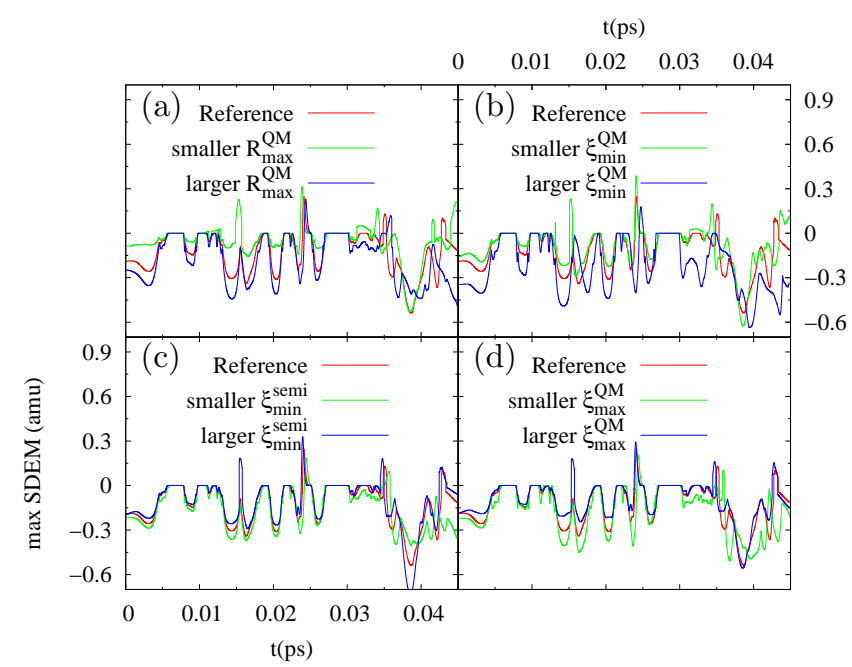

Figure 4: The SDEMs with the largest absolute value in $\mathrm{MM} / \mathrm{MM}$ simulations, showing the effect of changing the partition parameters $\left(R_{\max }^{\mathrm{QM}}, \xi_{\mathrm{min}}^{\mathrm{semi}}\right.$, $\left.\xi_{\min }^{\mathrm{QM}}, \xi_{\max }^{\mathrm{QM}}\right)$ on the SDEM. The reference curve is the $\xi$ full curve in Fig. 3(c). The partition parameters of other simulations are listed in the following: (a) $R_{\max }^{\mathrm{QM}}=3 \AA$ Aand $4.5 \AA$; (b) $\xi_{\min }^{\mathrm{QM}}=8.875$ Åand $9.125 \AA$; (c) $\xi_{\text {min }}^{\text {semi }}=7.875 \AA$ Åand $8.125 \AA$; (d) $\xi_{\max }^{\mathrm{QM}}=9.875 \AA$ Aand $10.125 \AA$.

Small SDEMs are needed to ensure that the SDAC dynamics is close to that of the real system.
The SDEM depends on $1 / v_{\alpha}, \partial V / \partial \lambda_{\beta}, \partial \lambda_{\beta} / \partial \xi_{\gamma}$ and $\partial \xi_{\gamma} / \partial v_{\alpha}$ according to Eq. (10). The third term is directly determined by the partition parameters $\left(R_{\max }^{\mathrm{QM}}, \xi_{\min }^{\mathrm{semi}}, \xi_{\min }^{\mathrm{QM}}, \xi_{\max }^{\mathrm{QM}}\right)$, and the second and fourth terms are indirectly affected. These terms do not change monotonically with respect to parameter changes. Fig. 4 demonstrate the changes in max SDEM induced by changes in the partition parameters, and none of the partition parameter changes can lead to a smaller max SDEM at all times. For example, a smaller $W^{\text {buf }}$ can be expected to lead to smaller SDEM, since there are less $\partial V / \partial \lambda_{\beta}$ terms which are observed to have the same sign in many situations. This can be achieved with smaller $R_{\max }^{\mathrm{QM}}, \xi_{\mathrm{min}}^{\mathrm{QM}}$ and larger $\xi_{\min }^{\mathrm{semi}}, \xi_{\max }^{\mathrm{QM}}$. Although in many time periods the corresponding curves in Fig. 4 are closer to zero than the reference, some of the peaks become higher due to the increase of $\partial \lambda_{\beta} / \partial \xi_{\gamma}$, which is another effect of having a smaller $W^{\text {buf }}$. It is therefore difficult to tune the size of the SDEM by changing partition parameters.

A more reliable way to reduce the size of the SDEM is to change the functional form of the criterion. I demonstrate this with the following $\xi^{\mathrm{v}}$ and $\xi^{\log \mathrm{v}}$ criteria:

$$
\begin{aligned}
\xi_{\alpha}^{\mathrm{v}} & =v_{\alpha}, \\
\xi_{\alpha}^{\log \mathrm{v}} & =\log _{10} v_{\alpha} .
\end{aligned}
$$

Fig. 5 plots the max SDEMs when using $\xi^{\text {full }}$, $\xi^{\log v}$ and $\xi^{v}$ as criteria. The initial direction of the PKA of these simulations are different from those in Fig. 3 to enhance the contrast. The partition parameters ensure that $W^{\text {buf }}=R_{\max }^{\mathrm{QM}}(4 \AA)$ for all simulations, so that the difference between the $\xi^{\mathrm{v}}$ and $\xi^{\log v}$ curves is entirely due to the different functional form.

The $\partial \xi_{\gamma} / \partial v_{\alpha}$ term of Eq. (10) for the curves in Fig. 5 are

$$
\begin{aligned}
\frac{\partial \xi_{\gamma}^{\text {full }}}{\partial v_{\alpha}} & =\frac{2 m_{\alpha} v_{\alpha} \delta_{\alpha \gamma}}{\left|V_{\alpha}\left(\sigma_{\alpha}^{x x}+\sigma_{\alpha}^{y y}+\sigma_{\alpha}^{z z}\right)\right| \ln 10} \\
\frac{\partial \xi_{\gamma}^{\mathrm{v}}}{\partial v_{\alpha}} & =\delta_{\alpha \gamma} \\
\frac{\partial \xi_{\gamma}^{\operatorname{logv}}}{\partial v_{\alpha}} & =\frac{\delta_{\alpha \gamma}}{v_{\alpha} \ln 10}
\end{aligned}
$$




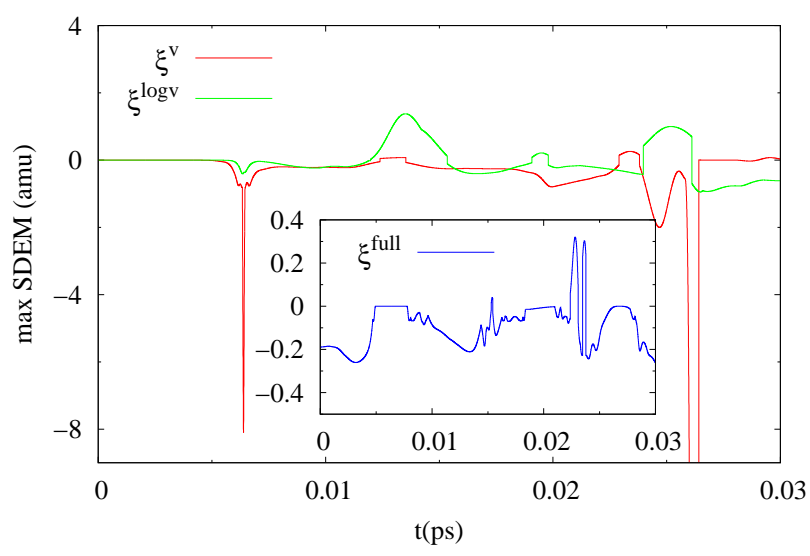

Figure 5: The SDEMs with the largest absolute value in MM/MM simulations using $\xi_{\alpha}^{\text {full }}, \xi_{\alpha}^{\mathrm{v}}$, and $\xi_{\alpha}^{\log v}$ as the criteria. For this figure, the initial velocity of the PKA is along the (001) direction. $R_{\mathrm{max}}^{\mathrm{QM}}=4 \AA$ for all simulations. The partition parameters of the $\xi^{\text {full }}$ simulation is the same as in Fig. 3. The partition parameters of the $\xi^{\mathrm{v}}$ simulation is $\xi_{\mathrm{min}}^{\mathrm{semi}}=0.1 v_{\mathrm{PKA}}^{\text {init }}, \xi_{\mathrm{min}}^{\mathrm{QM}}=0.55 v_{\mathrm{PKA}}^{\text {init }}$, $\xi_{\max }^{\mathrm{QM}}=v_{\mathrm{PKA}}^{\text {init }}$, where $v_{\mathrm{PKA}}^{\text {init }}$ is the initial speed of the PKA. The partition parameters of the $\xi^{\operatorname{logv}}$ simulation are $\xi_{\mathrm{min}}^{\text {semi }}=\log _{10}\left(0.1 v_{\mathrm{PKA}}^{\text {init }}\right), \quad \xi_{\mathrm{min}}^{\mathrm{QM}}=$ $\left[\log _{10}\left(0.1 v_{\text {PKA }}^{\text {init }}\right)+\log _{10}\left(v_{\text {PKA }}^{\text {init }}\right)\right] / 2$, and $\xi_{\max }^{\mathrm{QM}}=$ $\log _{10}\left(v_{\text {PKA }}^{\text {init }}\right)$.
Since $\partial \xi_{\alpha}^{\mathrm{v}} / \partial v_{\alpha}=1$, the $\xi^{\mathrm{v}}$ curve in Fig. 5 represents the changes of SDEM due to the other three terms in Eq. (10). The $\xi^{\mathrm{v}}$ curve has sharp valleys at about $0.006 \mathrm{ps}$ and $0.026 \mathrm{ps}$ which correspond to collisions between the PKA and other atoms, while $\xi^{\log v}$ curve does not have such features even though its functional form differs from $\xi^{\mathrm{v}}$ only by a simple logarithm. This is due to the difference in Eqs. (18) and (19), which behaves as $O(1)$ and $O\left(1 / v_{\alpha}\right)$ respectively. At about 0.006 ps and 0.026 ps, the atom corresponding to the max SDEM is the PKA, and the $\xi^{\log v}$ max SDEM is much smaller than the $\xi^{\mathrm{v}}$ one since the speed of the PKA is large. At other times (such as between $0.011 \mathrm{ps}$ and $0.015 \mathrm{ps}$ ), the atom corresponding to the max SDEM may not be the PKA, so the $\xi^{\log v} \max S$ DEM may become larger instead.

Since $\partial \xi_{\gamma}^{\text {full }} / \partial v_{\alpha}$ behaves as $O\left(v_{\alpha}\right)$ when $v_{\alpha}$ is small, and behaves as $O\left(1 / v_{\alpha}\right)$ when $v_{\alpha}$ is large, the $\xi^{\text {full }} \max$ SDEM is always small, making it a much better choice as the criterion than $\xi^{\mathrm{v}}$ and $\xi^{\log v}$ In the inset of Fig. 5, the $\xi^{\text {full }}$ curve is smal1 comparing to the atomic mass of Silicon at all times.

\subsubsection{Comparison of dynamics}

I use SDAC MM/MM simulations to check the agreement between the SDAC dynamics and the dynamics described by the 'higher-level' MM potential. The simulations are carried out in a $15 \times$ $15 \times 15$ supercell. The 2 outermost layers of atom$\mathrm{s}$ are fixed, ${ }^{4,7,9,10}$ and the next 4 layers of atoms are Langevin thermostated ${ }^{92}$ at $300 \mathrm{~K}$ following the setup in literature. ${ }^{4-15}$ This allows the excess energy introduced to the system by the PKA to dissipate in order to simulate the DD generation. The initial kinetic energy of the PKA is set to $0.5 \mathrm{keV}$, and the initial direction of the PKA is the same as in Sec. 3.2.1. The partition parameters are the same as in Fig. 3.

Fig. 6(a) plots the average temperature of the interior region (inside the temperature controlling layers) where the DD generation takes place. Although the temperature is not well-defined for the non-equilibrium process, its change over time can be used as a rough indicator of the rate of the dissipation of the excess energy. In the SDAC and $\mathrm{AC} M M / M M$ simulations, the dissipation of the 
excess energy is mainly determined by the 'lowerlevel' potential (SW), since the 'higher-level' potential (Tersoff/ZBL) only controls the interaction of atoms in the active regions. Fig. 6(a) agrees with this analysis: the difference between the SW and the Tersoff/ZBL curves can be seen clearly during $0.01 \sim 0.025 \mathrm{ps}$ and during $0.1 \sim 1 \mathrm{ps}$, and both the AC and the SDAC curves in these durations follow the SW curve closely as expected.

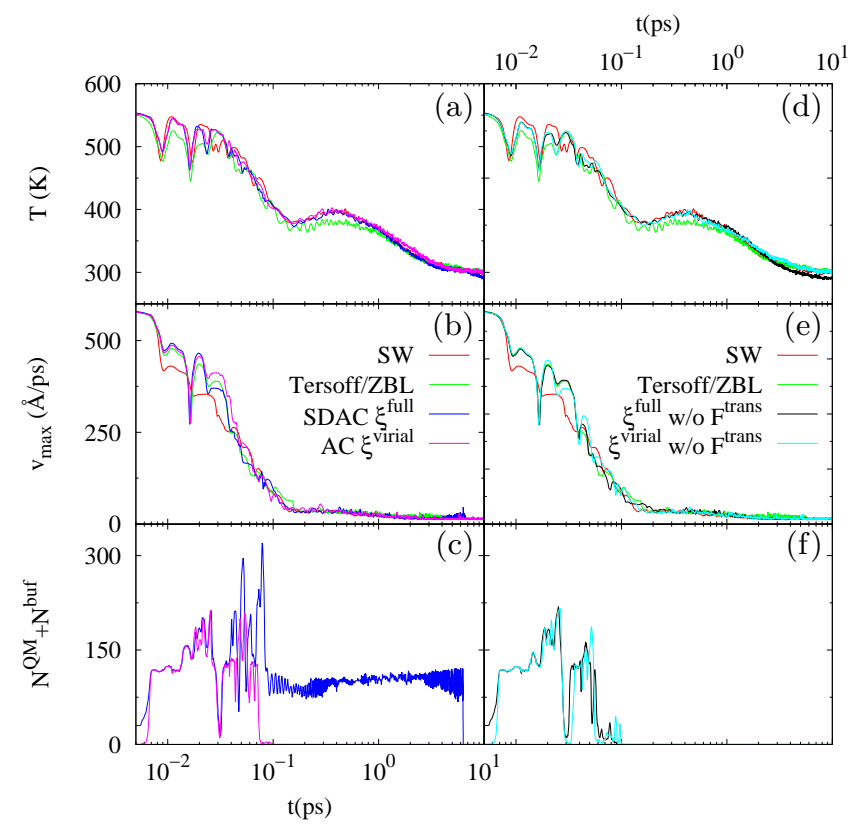

Figure 6: The temperature, the max atomic speed and the number of QM/buffer atoms of AC ( $\xi^{\text {virial }}$ ) and SDAC ( $\left.\xi^{\text {full }}\right)$ simulations. Results of MD simulations with SW and Tersoff/ZBL potentials are plotted for comparison. The temperature is obtained from the average kinetic energy of the nonthermostated atoms. Panels (a)-(c) plot the result$\mathrm{s}$ of simulations including transition forces, and panels (d)-(f) plot the results of simulations without transition forces.

The maximum speeds of all atoms are plotted in Fig. 6(b). The difference between the SW and the Tersoff/ZBL curves is the most obvious during $0.008 \sim 0.07 \mathrm{ps}$. Since the regions around fastmoving atoms are active regions in the SDAC simulation, the SDAC curve is close to the 'higherlevel' Tersoff/ZBL curve in this time duration, and the degree of the agreement between these curves indicate the quality of the adaptively determined partition of the system. The AC curve also follows the trend of the Tersoff/ZBL curve, since the fast- moving atoms about to collide with other atoms are also identified as active region centers.

Fig. 6(c) plots the number of QM/buffer atoms. The QM/buffer regions of the AC simulation vanish at about $0.1 \mathrm{ps}$, but those of the SDAC simulation continue to exist until about $6.3 \mathrm{ps}$, and the number of QM/buffer atoms oscillates rapidly. This is an artifact due to the inclusion of the transition forces of Eq. (6). The transition forces lead to an artificial heating of the buffer region, which affects the speed of the active region center through the interactions with the buffer atoms. The overall effect is that $\xi^{\text {full }}$ of the active region center does not drop below $\xi_{\text {min }}^{\text {semi }}$ for a long time. The heating of the buffer region also exists for the AC simulation, but the effect is not as significan$\mathrm{t}$ since its criterion does not depend on the speed. The small peak at about $6.3 \mathrm{ps}$ in the SDAC curve of Fig. 6(b) is also due to the artificial heating by transition forces.

As mentioned before, Hamiltonian corrections to transition forces are unavailable for the modSISPA AP-QM/MM method used in this paper. Pezeshki et al ${ }^{66}$ suggested that the transition forces should be discarded directly to avoid the geometry distortion and the artificial heating, and the system is coupled to a thermostat since the energy is no longer conserved. The results of the corresponding AC and SDAC simulations without transition forces are plotted in Fig. 6(d)-(f). The difference between Fig. 6(c) and (f) confirms that the long tail of the SDAC curve is due to transition forces. The heating of the buffer region is also evident by comparing the curves of Fig. 6(a) and (d) during $0.01 \sim 0.02$ ps. Without the transition forces, the SDAC max speed curve in Fig. 6(e) has a much better agreement with the 'higher-level' Tersoff/ZBL curve in the $0.008 \sim 0.07 \mathrm{ps}$ time duration than the curve in Fig. 6(b).

Amorphous disordered regions form in the DD generation process. $5,7,18,28,93-95$ Different properties are used in literature to identify the atom$\mathrm{S}$ in the disordered region, such as the atomic potential energies, ${ }^{4,7}$ the bond angles, ${ }^{5}$ the ring structures, ${ }^{15,28}$ the Lindemann spheres, ${ }^{9,30}$ and the time-average of atomic positions. ${ }^{10}$ The WignerSeitz defect analysis ${ }^{84}$ has been widely used as well, 6,11,14,21 even though it is less suitable for amorphous structures. I use the atomic potential 


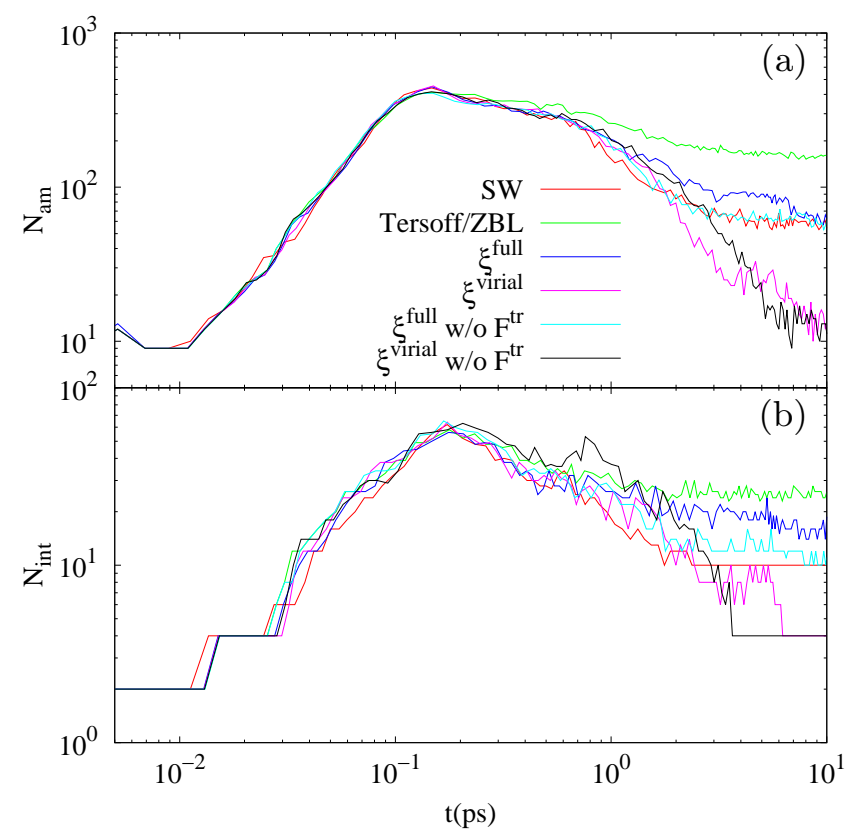

Figure 7: The number of atoms in amorphous regions (panel a) and the number of interstitial$\mathrm{s}$ (panel b) plotted with respect to the simulation time.

energy for simplicity, and atoms with potential energies greater than the crystalline average by 0.2 $\mathrm{eV}^{4,7}$ are identified as disordered atoms. All the atomic potential energies are calculated with the SW potential so that the results can be compared directly. I also use the Wigner-Seitz defect analysis to find interstitial and vacancy defects in the following.

Fig. 7 plots the number of interstitials $\left(N_{\text {int }}\right)$ in panel (a) and the number of disordered atoms $\left(N_{\mathrm{am}}\right)$ in panel (b). It shows that the defect generation finishes at about $0.15 \mathrm{ps}$, and most of this process is described by the 'higher-level' method in AC/SDAC simulations, since the QM/buffer regions exist at least for $0.1 \mathrm{ps}$ according to Fig. 6 . Fig. 7 confirms this as the curves of AC/SDAC simulations are close to the Tersoff/ZBL curve for the first $0.1 \mathrm{ps}$. After $0.1 \mathrm{ps}$, the dynamics is described by the 'lower-level' SW potential in the AC simulations and the SDAC simulation without transition forces, and the curves of these simulations in Fig. 7 slowly deviate from the Tersoff/ZBL curve.

Due to the unphysical heating of the buffer region by transition forces, the QM/buffer regions of the SDAC simulation with transition forces does not vanish until about $6.3 \mathrm{ps}$. The deviation of the $\xi^{\text {full }}$ curve from the Tersoff/ZBL curve is consequently not as much as other AC and SDAC curves after 0.1 ps. This is not an advantage, however, since other aspects of the dynamics (such as $v_{\max }$ ) become worse.

The number of defects stabilizes as the system cools down to $300 \mathrm{~K}$ at about 10 ps. For SDAC with/without transition forces, the numbers of defects left at $10 \mathrm{ps}$ are either in between that of the $\mathrm{SW}$ and the Tersoff/ZBL potentials or very close to that of the SW potential. For AC simulation$\mathrm{s}$, however, the numbers of defects left at $10 \mathrm{ps}$ are much less. This demonstrates the deficiency of the AC method in the simulation of DD generation. The AC method can only partition the system according to geometrical criteria. With the AC method, the collision of atoms is treated with the higher-level method only after atoms are very close to each other, and is treated with the lowerlevel method when atoms are not close enough (Fig. 2), so the description of the collision process is inconsistent. This affects the geometries of the defect clusters and leads to the much faster healing of damage. The entire collision process is described consistently by the higher-level method in SDAC simulations, and the resulting annealing behaviors in Fig. 7 are more reasonable.

Fig. 8 shows the geometries of the amorphous disordered regions in the simulations at $0.15 \mathrm{ps}$ and at $10 \mathrm{ps}$. The sizes of the amorphous regions are characterized by the radius of gyration ${ }^{5}(\langle d R\rangle$ in Fig. 8), which is defined as

$$
\langle d R\rangle=\sqrt{\frac{1}{N_{\mathrm{am}}}} \sum_{\alpha}^{\text {disordered }}\left|\vec{r}_{\alpha}-\vec{r}_{\mathrm{cm}}\right|^{2},
$$

with $\vec{r}_{\mathrm{cm}}=\frac{1}{N_{\mathrm{am}}} \sum_{\alpha}^{\text {disordered }} \vec{r}_{\alpha} \cdot\langle d x\rangle,\langle d y\rangle$, and $\langle d z\rangle$ are defined similarly. The aspect ratios $\langle d x\rangle /\langle d y\rangle$ and $\langle d x\rangle /\langle d z\rangle$ in Fig. 8 characterize the morphology of the disordered region.

At $0.15 \mathrm{ps}$, the front view of the disordered region of the SW simulation has a roughly elliptical shape which is rotated clockwise, and that of the Tersoff/ZBL simulation is roughly wedge-shaped with the narrow side pointing bottom left. The $\mathrm{SW}$ one is also larger in size and more extended in the $x$ direction comparing with that of the Ter- 


\begin{tabular}{|c|c|c|c|c|c|}
\hline $\begin{array}{l}\text { SW } \\
0.15 \mathrm{ps} \\
\langle d R\rangle=14.73 \mathrm{~A} \\
\frac{\langle d x\rangle}{\langle d y\rangle}=2.384 \\
\frac{\langle d x\rangle}{\langle d z\rangle}=1.756\end{array}$ & 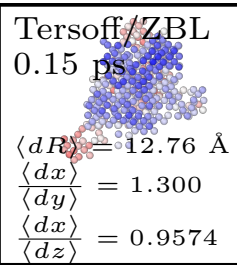 & $\begin{array}{c}\xi^{\text {full }} \\
0.15 \mathrm{ps} \\
{ }^{2} \\
\langle d R\rangle=12.40 \AA \\
\frac{\langle d x\rangle}{\langle d y\rangle}=1.461 \\
\frac{\langle d x\rangle}{\langle d z\rangle}=1.118\end{array}$ & $\begin{array}{c}\xi^{\text {virial }} \\
0.15 \text { ps } \\
\\
\langle d R\rangle^{3}=012.05 \AA \\
\frac{\langle d x\rangle}{\langle d y\rangle}=1.243 \\
\frac{\langle d x\rangle}{\langle d z\rangle}=1.064\end{array}$ & 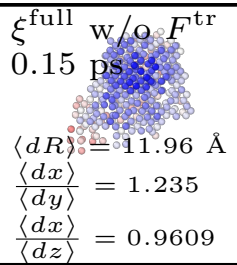 & $\begin{array}{c}\xi^{\mathrm{virial}} \mathrm{w} / \mathrm{O} F^{\mathrm{tr}} \\
0.15 \mathrm{ps} \\
\langle d R\rangle^{\circ=}=12.25 \AA \\
\frac{\langle d x\rangle}{\langle d y\rangle}=1.346 \\
\frac{\langle d x\rangle}{\langle d z\rangle}=1.070\end{array}$ \\
\hline $\begin{array}{l}\text { SW } \\
10 \text { ps } \\
\\
\langle d R\rangle=12.71 \AA \\
\frac{\langle d x\rangle}{\langle d y\rangle}=4.183 \\
\frac{\langle d x\rangle}{\langle d z\rangle}=1.361\end{array}$ & $\begin{array}{l}\text { Tersoff } \% \text { ZBL } \\
10 \mathrm{ps} \\
\langle d R\}^{*}=01.94 \AA \\
\frac{\langle d x\rangle}{\langle d y\rangle}=1.233 \\
\frac{\langle d x\rangle}{\langle d z\rangle}=0.8031\end{array}$ & $\begin{array}{l}\xi^{\text {full }} \\
10 \mathrm{ps} \\
\langle d R\rangle=10.08 \AA \\
\frac{\langle d x\rangle}{\langle d y\rangle}=1.621 \\
\frac{\langle d x\rangle}{\langle d z\rangle}=1.012\end{array}$ & $\begin{array}{l}\xi^{\text {virial }} \\
10 \mathrm{ps} \\
\langle d R\rangle=11.13 \AA \\
\frac{\langle d x\rangle}{\langle d y\rangle}=4.837 \\
\frac{\langle d x\rangle}{\langle d z\rangle}=1.950\end{array}$ & $\begin{array}{c}\xi^{\text {full }} \mathrm{w} / \mathrm{o} F^{\mathrm{tr}} \\
10 \mathrm{ps}{ }^{\circ} \\
\langle d R\rangle=8.644 \AA \\
\frac{\langle d x\rangle}{\langle d y\rangle}=0.8532 \\
\frac{\langle d x\rangle}{\langle d z\rangle}=0.7650\end{array}$ & $\begin{array}{l}\xi^{\text {virial }} \mathrm{w} / \mathrm{o} F^{\mathrm{tr}} \\
10 \mathrm{ps} \\
\langle d R\rangle=8.960 \AA \\
\frac{\langle d x\rangle}{\langle d y\rangle}=3.068 \\
\frac{\langle d x\rangle}{\langle d z\rangle}=1.086\end{array}$ \\
\hline
\end{tabular}

Figure 8: Front view ( $x z$ plane) snapshots of the amorphous pockets in the simulations. The atoms in the amorphous regions are identified by the atomic potential energy. The blue-white-red color coding represent the $y$ coordinate from small to large.

soff/ZBL simulation. All the AC and SDAC simulations result in disordered regions with similar shapes and sizes as in the Tersoff/ZBL simulation, showing that the AC and SDAC methods are able to correctly identify the active regions of the DD process and apply the 'higher-level' Tersoff/ZBL potential to these regions.

At 10 ps, most of the damage in the SW simulation is healed, and remaining disordered atoms are grouped in several smaller clusters with similar sizes. In the Tersoff/ZBL simulation, a large number of disordered atoms remains, and most of them belong to a large cluster. The dynamics of the annealing process for all AC/SDAC simulations is determined by the SW potential, but the resulting stabilized disordered regions have very differen$t$ shapes. The AC simulations end up with very few disordered atoms and does not resemble the results of both the SW and the Tersoff/ZBL simulations. For the SDAC simulations, the numbers of disordered atoms at 10 ps are almost the same as that of the SW simulation according to Fig. 7, but the geometry of the disordered region retains some of the characters of that of the Tersoff/ZBL simulation, since the disordered atoms are mostly gathered in a large cluster.

\subsection{SDAC QM/MM simulation of the DD generation in bulk Silicon}

In the following, I demonstrate the SDAC method with actual QM/MM simulations using DFTB and the SW potential as the QM and the MM method- s. The transition forces are neglected in the SDAC QM/MM simulation. The simulations are carried out with a $20 \times 20 \times 20$ supercell, and other aspects of the simulations are the same as in Sec. 3.2.2. An MD simulation with the SW potential and an (Tersoff/ZBL)/SW MM/MM simulation without transition forces are carried out for comparison. $\xi^{\text {full }}$ is used as the SDAC criterion for QM/MM and M$\mathrm{M} / \mathrm{MM}$ simulations, and the partition parameters are the same as in Sec. 3.2.2.

Fig. 9 plots the same quantities as in Fig. 6. A SDAC QM/MM simulation with transition forces is carried out for comparison, whose curves are plotted in the insets of Fig. 9, and such a simulation stops at about $0.12 \mathrm{ps}$ as the buffer regions begin to touch the temperature controlling layer. The temperature in the inset of Fig. 9(a) increases over time, showing that the QM/MM artificial heating due to transition forces is much worse than that of MM/MM comparing with Fig. 6(a). This signifies the large difference in the description of the interactions in the QM and MM methods, which determines the sizes of the transition forces through $\partial V / \partial \lambda_{\alpha}$. The SDEM in the QM/MM simulation is in the range of $[-2.5,0]$, which is about 10 times larger than the $\xi^{\text {full }}$ curve of Fig. 5, but still small comparing with the atomic mass of Si. By carrying out simulations without the SDEM, I find the effect of the SDEM on the result being much smaller than that of the transition forces.

The relative strengths of the QM and the MM interactions can be seen from the different behaviors of the curves in Fig. 9. The average temper- 


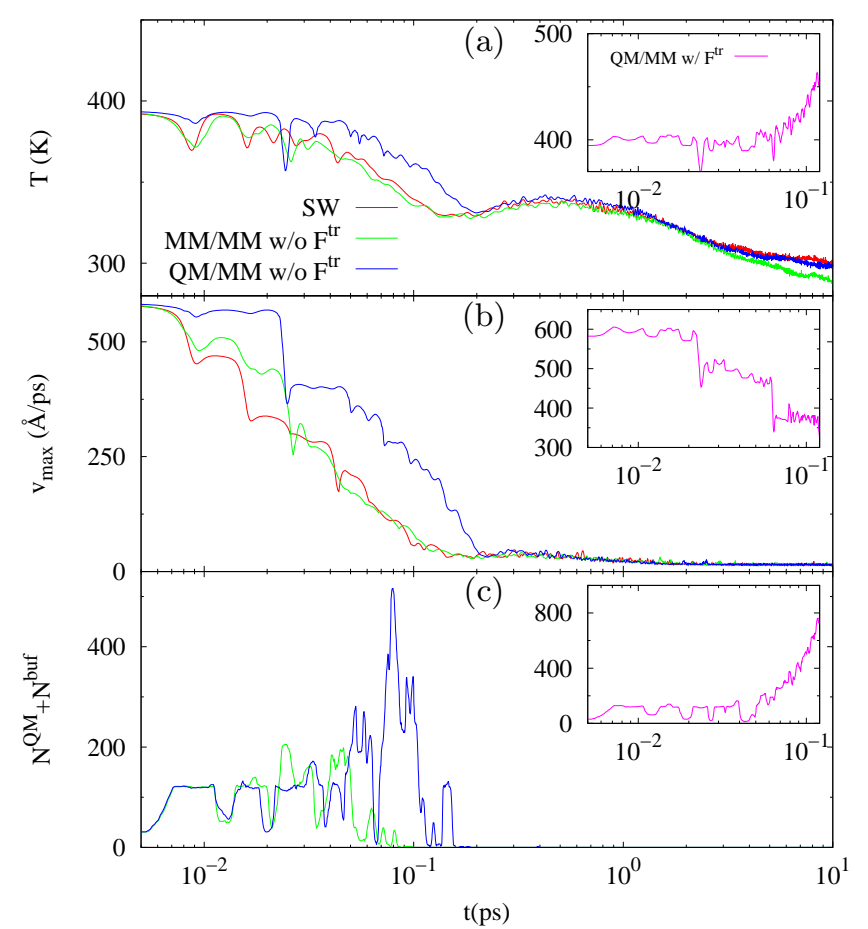

Figure 9: The temperature, the max atomic speed and the number of QM/buffer atoms of the SDAC QM/MM simulation without transition forces. Results of simulations with other methods are plotted for comparison.

atures in Fig. 9(a) show that the dissipation of the excess energy of the PKA is slower in QM/MM than in SW or in MM/MM, which suggests that the QM (DFTB) interaction is in general weaker than the MM interactions (both the SW and the Tersoff/ZBL potentials). This can also be seen from the $v_{\max }$ curves in Fig. 9(b), as the first dip of the QM/MM curve at about 0.009 ps is much shallower than the first dips of the SW and the M$\mathrm{M} / \mathrm{MM}$ curves. The magnitude of the MM/MM $v_{\max }$ change at about $0.024 \mathrm{ps}$ is close to that of the QM/MM curve, showing the effect of the ZBL short-range interaction.

Fig. 10 shows that the damaged region grows slower in QM/MM as the peaks of the QM/MM curves occur later than those of the SW and the MM/MM curves. This agrees with the recently reported machine learning results, ${ }^{14}$ where the number of interstitials reaches the maximum later than in simulations with SW or Tersoff potentials. The machine learning potential is also found to behave similarly as the SW potential for the annealing process in terms of $N_{\text {int }}{ }^{14}$ This corroborates the

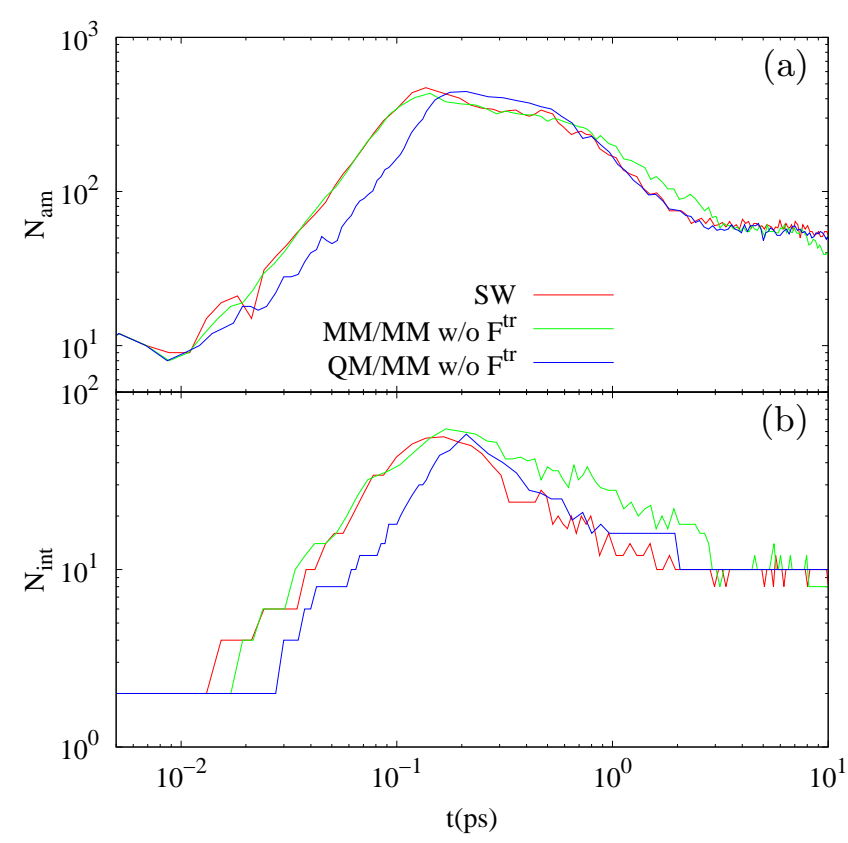

Figure 10: The number of atoms in amorphous regions (panel a), the number of interstitials (panel b) plotted against the simulation time.

validness of the QM/MM simulation of this paper, whose annealing stage is controlled by the SW potential according to Fig. 9(c).

More atoms are treated with the higher-level method in QM/MM than in MM/MM according to Fig. 9(c), indicating a larger disordered region in the QM/MM simulation. This is seen clearly in the snapshots of Fig. 11 where the disordered region generated in the QM/MM simulation is more extended in the $y$ and $z$ directions. The extended shape of the QM/MM disordered region and the separated small defect clusters after annealing (see color coding) also agrees with the machine learning results of Hamedani et al. ${ }^{14}$

\section{Conclusion}

In this paper, I extend the AC method of my previous work ${ }^{62}$ and develop an SDAC method to allow the active region centers in AP-QM/MM simulations to be determined on-the-fly according to speed-dependent properties, and I validate and demonstrate the method by applying it on the AP$\mathrm{MM} / \mathrm{MM}$ and AP-QM/MM simulations of the DD generation in bulk Si. AP-MM/MM simulations show that the SDAC method improves over the 


\begin{tabular}{|c|c|c|}
\hline $\begin{array}{l}\text { SW } \\
0.17 \mathrm{ps} \\
\\
\langle d R\rangle=12.82 \AA \\
\frac{\langle d x\rangle}{\langle d y\rangle}=0.9288 \\
\frac{\langle d x\rangle}{\langle d z\rangle}=1.287\end{array}$ & $\begin{array}{l}\mathrm{MM} / \mathrm{MM} \\
0.17 \mathrm{ps} \\
\\
\langle d R\rangle=11.97 \AA \\
\frac{\langle d x\rangle}{\langle d y\rangle}=1.248 \\
\frac{\langle d x\rangle}{\langle d z\rangle}=1.185\end{array}$ & $\begin{array}{l}\text { QM/M } \\
0.17 \mathrm{ps} \\
\langle d R\rangle=16.07 \AA \\
\frac{\langle d x\rangle}{\langle d y\rangle}=0.5079 \\
\frac{\langle d x\rangle}{\langle d z\rangle}=0.7225\end{array}$ \\
\hline $\begin{array}{l}\mathrm{SW} \\
10 \mathrm{ps} \\
\langle d R\rangle=11.11 \AA \\
\frac{\langle d x\rangle}{\langle d y\rangle}=1.272 \\
\frac{\langle d x\rangle}{\langle d z\rangle}=1.688\end{array}$ & $\begin{array}{l}\mathrm{MM} / \mathrm{MM} \\
10 \mathrm{ps} \\
\langle d R\rangle=10.49 \AA \\
\frac{\langle d x\rangle}{\langle d y\rangle}=1.774 \\
\frac{\langle d x\rangle}{\langle d z\rangle}=0.8459\end{array}$ & $\begin{array}{l}\text { QM/MAM } \\
10 \mathrm{ps} \\
\langle d R\rangle=15.05 \AA \\
\frac{\langle d x\rangle}{\langle d y\rangle}=0.4860 \\
\frac{\langle d x\rangle}{\langle d z\rangle}=0.8897\end{array}$ \\
\hline
\end{tabular}

Figure 11: Front view ( $x z$ plane) snapshots of the amorphous pockets in the simulations. The atom$\mathrm{s}$ in the amorphous regions are identified by the atomic potential energy. The $\mathrm{MM} / \mathrm{MM}$ and the QM/MM simulations are carried out without transition forces. The blue-white-red color coding represent the $y$ coordinate from small to large.

previous AC method, since the SDAC dynamics, number of defects, and the shape of the disordered region agrees better with those of the 'higherlevel' MM method than the AC results. Although the motivation for developing the SDAC method is for the simulation of the DD generation, the method is applicable to general problems in which active regions may occur or vanish during the simulation and have a speed dependence, such as the dynamics of shockwaves or collisions.

The SDAC PES is speed-dependent, and the usual EOM in MD becomes inapplicable. I develop an energy-conserving SDAC EOM, which is differen$\mathrm{t}$ from Newton's second law by the SDEM term. The time integration of the non-standard EOM is carried out with the EPS algorithm. The dynamics of the SDAC QM/MM system can be expected to be close to that of the real system when the SDEM is much smaller than atomic masses. I show that the size of the SDEM can be effectively tuned by the functional form of the SDAC criterion property, which allows the SDAC method to be applicable in general systems.

The transition forces in AP-QM/MM lead to geometry distortions and artificial heating. ${ }^{55,66,67}$ The same effect is observed in the SDAC simulations, and is found to be significant when the higher-level and lower-level methods of QM/MM differ a lot. I find that discarding the transition forces ${ }^{66}$ in SDAC simulations leads to obvious improvements to the simulations, although this may not remove all the artifacts since the simulated system is not fully thermostated.

Although the machine-learning potential by Hamedani, et al $^{14}$ is an important advancemen$t$ of simulation methods of the DD. it is still worthwhile to develop an QM/MM method that combines the accuracy and explicit treatment of electrons of QM methods and the efficiency of classical MM potentials as an alternative way to simulate the DD process. The black box nature of the machine-learning potential makes it difficult to estimate the accuracy in cases beyond its training set. The form of the machine-learning potential remains classical and does not treat electrons explicitly, so it is not clear whether it is able to fully describe the influence of electron-phonon coupling and of the disturbance of the electronic structure by fast-moving recoil ions. ${ }^{91}$ Although SDAC simulations have to use a ground-state QM method and are still missing non-adiabatic effects, the SDAC method provides a framework on which improvements that allow non-adiabatic molecular dynamics (NAMD) can be made, considering excited-state QM methods have been successfully applied in regular QM/MM. ${ }^{96-104}$ The preliminary SDAC QM/MM simulation presented in this work is able to reproduce many of the characteristics of the machine-learning results, despite remaining issues such as the treatment of transition forces and the SDEM term.

\section{Acknowledgements}

Acknowledgement The author is supported by the National Natural Science Foundation of China grant No. 11804314, and by Science Challenge Project, No. TZ2016003. 


\section{A EOM derived from La- grangian dynamics}

The Lagrangian EOM that conserves the Hamiltonian Eq. (8) is

$$
\begin{aligned}
m_{\alpha} \vec{a}_{\alpha}=-\nabla_{\vec{r}_{\alpha}} V+\sum_{\beta} & \left(\nabla_{\vec{r}_{\beta}} \cdot \nabla_{\vec{v}_{\alpha}} V\right) \vec{v}_{\beta} \\
& +\sum_{\beta}\left(\nabla_{\vec{v}_{\beta}} \cdot \nabla_{\vec{v}_{\alpha}}\right) \vec{a}_{\beta} .
\end{aligned}
$$

The speed-dependence of Eq. (21) is more complicated than that of Eq. (9). Since all atoms are coupled together in Eq. (21), it is difficult to estimate the deviation of the dynamics described by Eq. (21) from that of the real system.

\section{B Details of the EPS algorithm}

The original phase space at the initial time is $\left(\left\{\vec{r}_{\alpha}^{(0)}\right\},\left\{\vec{v}_{\alpha}^{(0)}\right\}\right)$, in which I use velocities instead of momenta for simpler notation. In the EPS algorithm, the original phase space is duplicated to form an extended phase space $\left(\left\{\vec{r}_{\alpha}\right\},\left\{\vec{v}_{\alpha}\right\},\left\{\tilde{\vec{r}}_{\alpha}\right\},\left\{\tilde{\vec{v}}_{\alpha}\right\}\right)$. The time integration is performed in the extended phase space, and the original phase space of a certain time step is obtained from the extended phase space by projection. One can choose to carry out the time integration entirely in the extended phase space, or project back to the original phase space and form the extended phase space with the projected values after a few steps. The EPS time propagation operator that run in the extended phase space for $k$ time steps can be written as: ${ }^{71}$

$$
\Psi^{(k)}=\hat{P} \circ\left[\hat{M}_{2} \hat{R} \hat{\tilde{V}} \hat{\tilde{R}} \hat{V} \hat{M}_{1} \hat{V} \hat{\tilde{R}} \hat{\tilde{V}} \hat{R}\right]^{k} \circ \hat{C}
$$

so that

$$
\Psi^{(k)}\left(\left\{\vec{r}_{\alpha}^{(i)}\right\},\left\{\vec{v}_{\alpha}^{(i)}\right\}\right)=\left(\left\{\vec{r}_{\alpha}^{(i+k)}\right\},\left\{\vec{v}_{\alpha}^{(i+k)}\right\}\right) .
$$

The propagators $\hat{R}, \hat{V}, \tilde{\hat{R}}$, and $\tilde{\hat{V}}$ of Eq. (22) perform update to $\left\{\vec{r}_{\alpha}\right\},\left\{\tilde{\vec{r}}_{\alpha}\right\},\left\{\vec{v}_{\alpha}\right\}$, and $\left\{\tilde{\vec{v}}_{\alpha}\right\}$ re- spectively:

$$
\begin{array}{ll}
\hat{R}: & \vec{r}_{\alpha} \leftarrow \vec{r}_{\alpha}+(\Delta t / 2) \vec{v}_{\alpha}, \\
\hat{V}: & \vec{v}_{\alpha} \leftarrow \vec{v}_{\alpha}+(\Delta t / 2) \vec{a}_{\alpha}\left(\vec{r}_{\alpha}, \tilde{\vec{v}}_{\alpha}\right), \\
\hat{\tilde{R}}: & \tilde{\vec{r}}_{\alpha} \leftarrow \tilde{\vec{r}}_{\alpha}+(\Delta t / 2) \tilde{\vec{v}}_{\alpha}, \\
\hat{\tilde{V}}: & \tilde{\vec{v}}_{\alpha} \leftarrow \tilde{\vec{v}}_{\alpha}+(\Delta t / 2) \vec{a}_{\alpha}\left(\tilde{\vec{r}}_{\alpha}, \vec{v}_{\alpha}\right),
\end{array}
$$

where $\Delta t$ is the size of the time step.

$\hat{C}$ of Eq. (22) is the operator that extend the original phase space into the extended phase space:

$$
\hat{C}\left(\left\{\vec{r}_{\alpha}^{(i)}\right\},\left\{\vec{v}_{\alpha}^{(i)}\right\}\right)=\left(\left\{\vec{r}_{\alpha}^{(i)}\right\},\left\{\vec{v}_{\alpha}^{(i)}\right\},\left\{\vec{r}_{\alpha}^{(i)}\right\},\left\{\vec{v}_{\alpha}^{(i)}\right\}\right)
$$

The mixing operators $\hat{M}_{n}(n=1,2)$ of Eq. (22) mixes the two parts of the extended phase space to improve the stability of the algorithm:

$$
\begin{aligned}
& \hat{M}_{n}\left(\left\{\vec{r}_{\alpha}\right\},\left\{\vec{v}_{\alpha}\right\},\left\{\tilde{\vec{r}}_{\alpha}\right\},\left\{\tilde{\vec{v}}_{\alpha}\right\}\right)= \\
& \quad\left(\left\{a_{n} \vec{r}_{\alpha}+\left(1-a_{n}\right) \tilde{\vec{r}}_{\alpha}\right\},\left\{b_{n} \vec{v}_{\alpha}+\left(1-b_{n}\right) \tilde{\overrightarrow{\vec{v}}}_{\alpha}\right\},\right. \\
& \left.\left.\left\{\left(1-a_{n}\right) \vec{r}_{\alpha}+a_{n} \tilde{\vec{r}}_{\alpha}\right)\right\},\left\{\left(1-b_{n}\right) \vec{v}_{\alpha}+b_{n} \tilde{\vec{v}}_{\alpha}\right\}\right) .
\end{aligned}
$$

The projection operator $\hat{P}$ of Eq. (22) projects the extended phase space to the original phase space:

$$
\begin{aligned}
& \hat{P}\left(\left\{\vec{r}_{\alpha}\right\},\left\{\vec{v}_{\alpha}\right\},\left\{\tilde{\vec{r}}_{\alpha}\right\},\left\{\tilde{\vec{v}}_{\alpha}\right\}\right)= \\
& \quad\left(\left\{a \vec{r}_{\alpha}+(1-a) \tilde{\vec{r}}_{\alpha}\right\},\left\{b \vec{v}_{\alpha}+(1-b) \tilde{\vec{v}}_{\alpha}\right\}\right) .
\end{aligned}
$$

Different choices of the mixing and projection parameters of the EPS algorithm are available in the literature, ${ }^{71-73}$ but they do not work well for the SDAC simulations and become unstable when the time step is larger than about $10^{-5} \mathrm{ps}$. This is due to that the QM/MM partitions of the two parts of the EPS may become different during the internal steps of the algorithm. I use $a_{1}=a_{2}=1 / 2$, $b_{1}=b_{2}=1 / 2, a=b=1 / 2$ in this paper similar to Luo, et al. ${ }^{74}$ Although the strong coupling between the two parts of the EPS by these parameters is considered undesirable in the original EP$S$ paper, ${ }^{71}$ these parameters allow the use of time steps much larger than $10^{-5} \mathrm{ps}$, which is needed for practical simulations.

The most computationally expensive step in the time-integration is the evaluation of the potential energy. The computational cost of the EPS algo- 
rithm per time step is therefore about 4 times larger than that of the velocity Verlet algorithm.

\section{References}

(1) Srour, J. R.; Palko, J. W. IEEE Trans. Nucl. Sci. 2013, 60, 1740.

(2) Srour, J. R.; Palko, J. W. 2013 IEEE nuclear and space radiation effects conference short course notebook; 2013.

(3) Nordlund, K.; Zinkle, S. J.; Sand, A. E.; Granberg, F.; Averback, R. S.; Stoller, R. E.; Suzudo, T.; Malerba, L.; Banhart, F.; Weber, W. J.; Willaime, F.; Dudarev, S. L.; Simeone, D. J. Nucl. Mater 2018, 512, 450.

(4) Caturla, M.-J.; Díaz de la Rubia, T.; Marqués, L. A.; Gilmer, G. H. Phys. Rev. B 1996, 54, 16683.

(5) Palko, J. W.; Srour, J. R. IEEE Trans. Nucl. Sci. 2008, 55, 2992.

(6) Backman, M.; Toulemonde, M.; Pakarinen, O. H.; Juslin, N.; Djurabekova, F.; Nordlund, K.; Debelle, A.; Weber, W. J. Comput. Mater. Sci. 2013, 67, 261.

(7) Diaz de la Rubia, T.; Gilmer, G. H. Phys. Rev. Lett. 1995, 74, 2507.

(8) Diaz de la Rubia, T.; Soneda, N.; Caturla, M. J.; Alonso, E. A. J. Nucl. Mater. 1997, 251, 13.

(9) Hobler, G.; Otto, G. Nucl. Instrum. Meth. Phys. Res. B 2003, 206, 81.

(10) Santos, I.; Marqués, L. A.; Pelaz, L.; López, P.; Aboy, M.; Barbolla, J. Mater. Sci. Eng. B 2005, 124, 372.

(11) Buchan, J. T.; Robinson, M.; Christie, H. J.; Roach, D. L.; Ross, D. K.; Marks, N. A. J. Appl. Phys. 2015, 117, 245901.

(12) Lehtinen, O.; Naydenov, B.; Börner, P.; Melentjevic, K.; Müller, C.; McGuinness, L. P.; Pezzagna, S.; Meijer, J.; Kaiser, U.; Jelezko, F. Phys. Rev. B 2016, 93, 035202.
(13) Liu, J.; Muíños, H. V.; Nordlund, K.; Djurabekova, F. Appl. Surf. Sci. 2020, 527, 146495.

(14) Hamedani, A.; Byggmästar, J.; Djurabekova, F.; Alahyarizadeh, G.; Ghaderi, R.; Minuchehr, A.; Nordlund, K. Mater. Res. Lett. 2020, 8, 364.

(15) Foiles, S. M. Nucl. Instrum. Meth. Phys. Res. B 2007, 255, 101.

(16) Myers, S. M.; Cooper, P. J.; Wampler, W. R. J. Appl. Phys. 2008, 104, 044507.

(17) Hull, R., Ed. Properties of crystalline silicon; INSPEC, Institution of Electrical Engineers, 1999.

(18) Hobler, G.; Otto, G. Mater. Sci. Semicond. Process. 2003, 6, 1.

(19) Bukonte, L.; Djurabekova, F.; Samela, J.; Nordlund, K.; Norris, S. A.; Aziz, M. J. Nucl. Instrum. Meth. Phys. Res. B 2013, 297, 23.

(20) Dudarev, S. L. Annu. Rev. Mater. Res. 2013, 43,35 .

(21) Beck, M. J.; Schrimpf, R. D.; Fleetwood, D. M.; Pantelides, S. T. Phys. Rev. Lett. 2008, 100, 185502.

(22) Lim, A.; Foulkes, W. M. C.; Horsfield, A. P.; Mason, D. R.; Schleife, A.; Draeger, E. W.; Correa, A. A. Phys. Rev. Lett. 2016, 116, 043201.

(23) Holmström, E.; Nordlund, K.; Hakala, M. Phys. Rev. B 2010, 82, 104111.

(24) Nordlund, K.; Ghaly, M.; Averback, R. S.; Caturla, M.; Diaz de la Rubia, T.; Tarus, J. Phys. Rev. B 1998, 57, 7556.

(25) Díaz de la Rubia, T. Annu. Rev. Mater. Sci. 1996, 26, 613.

(26) Nord, J.; Nordlund, K.; Keinonen, J. Phys. Rev. B 2002, 65, 165329.

(27) Peltola, J.; Nordlund, K.; Keinonen, J. Nucl. Instrum. Meth. Phys. Res. B 2002, 195, 269. 
(28) Marqués, L. A.; Pelaz, L.; Aboy, M.; Enríquez, L.; Barbolla, J. Phys. Rev. Lett. 2003, 91, 135504.

(29) Santos, I.; Marqués, L. A.; Pelaz, L.; López, P. Spanish conference on electron devices; 2007; p 37.

(30) Borodin, V. A. Nucl. Instrum. Meth. Phys. Res. B 2012, 282, 33 .

(31) Ridgway, M. C. et al. Phys. Rev. Lett. 2013, 110, 245502.

(32) Debelle, A.; Backman, M.; Thomé, L.; Nordlund, K.; Djurabekova, F.; Weber, W. J.; Monnet, I.; Pakarinen, O. H.; Garrido, F.; Paumier, F. Nucl. Instrum. Meth. Phys. Res. B 2014, 326, 326.

(33) Jiménez, F.; Ortiz, C. J. Comput. Mater. Sci. 2016, 113, 178.

(34) Nordlund, K.; Djurabekova, F.; Hobler, G. Phys. Rev. B 2016, 94, 214109.

(35) Nordlund, K.; Zinkle, S. J.; Sand, A. E.; Granberg, F.; Averback, R. S.; Stoller, R.; Suzudo, T.; Malerba, L.; Banhart, F.; Weber, W. J.; Willaime, F.; Dudarev, S. L.; Simeone, D. Nat. Comm. 2018, 9, 1084.

(36) Hohenberg, P.; Kohn, W. Phys. Rev. 1964, 136, B864.

(37) Kohn, W.; Sham, L. J. Phys. Rev. 1965, 140, A1133.

(38) Fiolhais, C., Nogueira, F., Marques, M., Eds. A primer in density functional theory; Lecture notes in physics; Springer: Berlin, 2003.

(39) Robinson, M. T.; Torrens, I. M. Phys. Rev. B 1974, 9, 5008 .

(40) Norgett, M. J.; Robinson, M. T.; Torrens, I. M. Nucl. Eng. Design 1975, 33, 50.

(41) Ziegler, J. F.; Biersack, J. P.; Littmark, U. The stopping and ranges of ions in solids; Pergamon Press: New York, 1985.
(42) Ziegler, J. F.; Biersack, J. P.; Ziegler, M. D. SRIM - the stopping and range of ions in matter; SRIM Co., 2008.

(43) Weber, W. J.; Duffy, D. M.; Thomé, L.; Zhang, Y. Curr. Opin. Solid State Mater. Sci. 2015, 19, 1 .

(44) Warshel, A.; Levitt, M. J. Mol. Biol. 1976, 103, 227.

(45) Lin, H.; Truhlar, D. G. Theor. Chem. Acc. 2007, 117, 185.

(46) Senn, H. M.; Thiel, W. Angew. Chem. Int. Ed. 2009, 48, 1198.

(47) Bernstein, N.; Kermode, J. R.; Csányi, G. Rep. Prog. Phys. 2009, 72, 026501.

(48) Bulo, R. E.; Michel, C.; Fleurat-Lessard, P.; Sautet, P. J. Chem. Theory Comput. 2013, 9, 5567.

(49) Zheng, M.; Waller, M. P. WIREs Comput. Mol. Sci. 2016, 6, 369.

(50) Duster, A. W.; Wang, C.-H.; Garza, C. M.; Miller, D. E.; Lin, H. WIREs Comput. Mol. Sci. 2017, 7, e1310.

(51) Kerdcharoen, K. R., T. Liedl; Rode, B. M. Chem. Phys. 1996, 211, 313.

(52) Kerdcharoen, T.; Morokuma, K. Chem. Phys. Lett. 2002, 355, 257.

(53) Csányi, G.; Albaret, T.; Payne, M. C.; de Vita, A. Phys. Rev. Lett. 2004, 93, 175503.

(54) Heyden, A.; Lin, H.; Truhlar, D. G. J. Phys. Chem. B 2007, 111, 2231.

(55) Bulo, R. E.; Ensing, B.; Sikkema, J.; Visscher, L. J. Chem. Theory Comput. 2009, 5, 2212.

(56) Nielsen, S. O.; Bulo, R. E.; Moore, P. B.; Ensing, B. Phys. Chem. Chem. Phys. 2010, 12,12401 .

(57) Watanabe, H. C.; Kubař, T.; Elstner, M. J. Chem. Theory Comput. 2014, 10, 4242. 
(58) Field, M. J. J. Chem. Theory Comput. 2017, 13, 2342.

(59) Watanabe, H. Molecules 2018, 23, 1882.

(60) Chen, H.; Liao, M.; Wang, H.; Wang, Y; Zhang, L. Comput. Methods Appl. Mech. Eng. 2019, 354, 351.

(61) Wang, Y.; Chen, H.; Liao, M.; Ortner, C.; Wang, H.; Zhang, L. arXiv: 2007.05260.

(62) Yang, Z.-H. Phys. Chem. Chem. Phys. 2020, 22, 19307.

(63) Rode, B. M.; Hofer, T. S.; Randolf, B. R.; Schwenk, C. F.; Xenides, D.; Vchirawongkwin, V. Theor. Chem. Acc. 2006, 115, 77.

(64) Bernstein, N.; Várnai, C.; Solt, I.; Winfield, S. A.; Payne, M. C.; Simon, I.; Fuxreiter, M.; Csányi, G. Phys. Chem. Chem. Phys. 2012, 14, 646.

(65) Thornton, S. T.; Marion, J. B. Classical dynamics of particles and systems; Brooks/Cole, 2004.

(66) Pezeshki, S.; Davis, C.; Heyden, A.; Lin, H. J. Chem. Theory Comput. 2014, 10, 4765.

(67) Boereboom, J. M.; Potestio, R.; Donadio, D.; Bulo, R. E. J. Chem. Theory Comput. 2016, 12, 3441.

(68) Yang, Z.-H. Phys. Chem. Chem. Phys. 2020, 22, 17987.

(69) Verlet, L. Phys. Rev. 1967, 159, 98.

(70) Donnelly, D.; Rogers, E. Am. J. Phys. 2005, $73,938$.

(71) Pihajoki, P. Celest. Mech. Dyn. Astr. 2015, 121, 211.

(72) Tao, M. Phys. Rev. E 2016, 94, 043303.

(73) Liu, L.; Wu, X.; Huang, G.; Liu, F. Mon. Not. R. Astron. Soc. 2016, 459, 1968.

(74) Luo, J.; Wu, X.; Huang, G.; Liu, F. Astrophys. J. 2017, 834, 64.
(75) Strang, G. SIAM J. Numer. Anal. 1968, 5, 506.

(76) Porezag, D.; Frauenheim, T.; Köhler, T.; Seifert, G.; Kaschner, R. Phys. Rev. B 1995, $51,12947$.

(77) Elstner, M.; Porezag, D.; Jungnickel, G.; Elsner, J.; Haugk, M.; Frauenheim, T.; Suhai, S.; Seifert, G. Phys. Rev. B 1998, 58, 7260 .

(78) Frauenheim, T.; Seifert, G.; Elstner, M.; Hajnal, Z.; Jungnickel, G.; Porezag, D.; Suhai, S.; Scholz, R. Phys. Stat. Sol. (b) 2000, 217, 41.

(79) Stillinger, F. H.; Weber, T. A. Phys. Rev. B $1985,31,5262$.

(80) Tersoff, J. Phys. Rev. B 1988, 37, 6991.

(81) Plimpton, S. J. J. Comput. Phys. 1995, 117, 1.

(82) http:// lammps.sandia.gov.

(83) Aradi, B.; Hourahine, B.; Frauenheim, T. J. Phys. Chem. A 2007, 111, 5678.

(84) Stukowski, A. Modelling Simul. Mater. Sci. Eng. 2010, 18, 015012.

(85) Elliott, R. S. Efficient 'universal' shifted Lennard-Jones model for all KIM API supported species developed by Elliott and Akerson (2015) v003. 2018; doi:10.25950/962b4967.

(86) Elliott, R. S. Efficient multi-species Lennard-Jones model with truncated or shifted cutoff v003. 2018; doi: $10.25950 /$ ac258694.

(87) Tadmor, E. B.; Elliott, R. S.; Sethna, J. P.; Miller, R. E.; Becker, C. A. JOM 2011, 63, 17.

(88) Elliott, R. S.; Tadmor, E. B. Knowledgebase of Interatomic Models (KIM) Application Programming Interface (API). 2011; doi:10.25950/ff8f563a. 
(89) Keinonen, J.; Arstila, K.; Tikkanen, P. Appl. Phys. Lett. 1992, 60, 628.

(90) Race, C. P.; Mason, D. R.; Finnis, M. W.; Foulkes, W. M. C.; Horsfield, A. P.; Sutton, A. P. Rep. Prog. Phys. 2010, 73, 116501 .

(91) Lan, M.; Yang, Z.-H.; Wang, X. Comput. Mater. Sci. 2020, 179, 109697.

(92) Schneider, T.; Stoll, E. Phys. Rev. B 1978, 17, 1302.

(93) Ruault, M. O.; Chaumont, J.; Penisson, J. M.; Bourret, A. Philos. Mag. A 1984, $50,667$.

(94) Holland, O. W.; White, C. W.; ElGhor, M. K.; Budai, J. D. J. Appl. Phys. 1990, 68, 2081.

(95) Donnelly, S. E.; Birtcher, R. C.; Vishnyakov, V. M.; Carter, G. Appl. Phys. Lett. 2003, $82,1860$.

(96) Röhrig, U. F.; Frank, I.; Hutter, J.; Laio, A.; VandeVondele, J.; Rothlisberger, U. ChemPhysChem 2003, 4, 1177.

(97) Moret, M.-E.; Tapavicza, E.; Guidoni, L.; Röhrig, U. F.; Sulpizi, M.; Tavernelli, I.; Rothlisberger, U. CHIMIA 2005, 59, 493.

(98) Virshup, A. M.; Punwong, C.; Pogorelov, T. V.; Lindquist, B. A.; Ko, C.; Martínez, T. J. J. Phys. Chem. B 2009, 113, 3280 .

(99) Kubař, T.; Elstner, M. J. Phys. Chem. B 2010, 114, 11221.

(100) Woiczikowski, P. B.; Steinbrecher, T.; Kubař, T.; Elstner, M. J. Phys. Chem. B 2011, 115, 9846.

(101) Steindal, A. H.; Rund, K.; Frediani, L.; Aidas, K.; Kongsted, J. J. Phys. Chem. B 2011, 115, 3027.

(102) Tavernelli, I.; Curchod, B. F. E.; Rothlisberger, U. Chem. Phys. 2011, 391, 101.
(103) Liang, W.; Chapman, C. T.; Ding, F.; Li, X. J. Phys. Chem. A 2012, 116, 1884.

(104) Kubař, T.; Elstner, M. Phys. Chem. Chem. Phys. 2013, 15, 5794. 